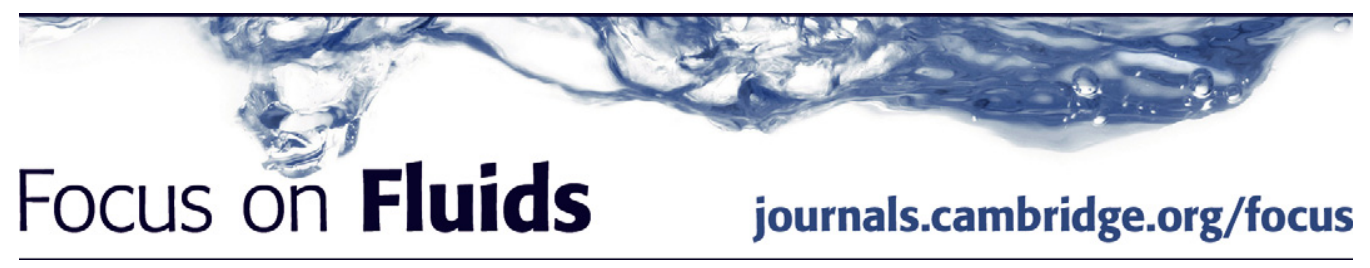

\title{
Multiphase flow in porous media
}

\author{
J. J. L. Higdon $\dagger$ \\ Department of Chemical and Biomolecular Engineering, \\ University of Illinois Urbana-Champaign, Urbana, IL 61801, USA
}

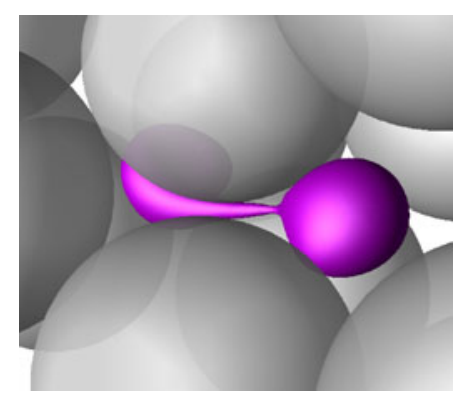

\begin{abstract}
Multiphase flows in porous media represent fluid dynamics problems of great complexity involving a wide range of physical phenomena. These flows have attracted the attention of an impressive group of renowned researchers and have spawned a number of classic problems in fluid dynamics. These multiphase flows are perhaps best known for their importance in oil recovery from petroleum reservoirs, but they also find application in novel areas such as hydrofracturing for natural gas recovery. In a recent article, Zinchenko \& Davis (J. Fluid Mech. 2013, vol. 725, pp. 611-663) present computational simulations that break new ground in the study of emulsions flowing through porous media. These simulations provide sufficient scale to capture the disordered motion and complex break-up patterns of individual droplets while providing sufficient statistical samples for estimating meaningful macroscopic properties of technical interest.
\end{abstract}

Key words: drops and bubbles, emulsions, porous media

\section{Introduction}

Multiphase flow in porous media is a subject of great complexity with a long, rich history in the field of fluid mechanics. This is a subject with important technical applications, most notably in oil recovery from petroleum reservoirs. Single-phase fluid flow through a porous medium is well characterized by Darcy's law, and the main features of the subject have been well understood for 150 years. By contrast, modelling of multiphase flow remains an enormous technical challenge. A wide gap exists between the model equations used in industrial application and a fundamental understanding of the detailed microscale physics. In the petroleum industry and in other technical applications, transport is modelled by postulating a multiphase generalization of Darcy's law. Here, distinct pressures are defined in each constituent phase with the difference, called capillary pressure, determined by the interfacial

$†$ Email address for correspondence: jhigdon@uiuc.edu 
tension, micropore geometry and surface chemistry of the solid medium. For flow rates, a relative permeability is defined relating the volume flow rate of each fluid to its pressure gradient. The prediction of the relative permeability as a function of material properties, morphology and saturation (relative volume fraction of a fluid phase) is a primary challenge for researchers in the petroleum industry. Classic reviews on multiphase flow in porous media have been given by Wooding \& Morel-Seytoux (1976) and Adler \& Brenner (1988).

It has often been said that all models are wrong, but some are useful, and this certainly holds true in the application of Darcy's law with relative permeabilities to multiphase flow. In the first instance, Darcy's law is linear, while the true governing equation is nonlinear as established by experiment and microscale analyses. Owing to the surface tension, the fluid flow is often shear thinning even when the constituent fluids are Newtonian. The flow may lead to local jamming when the pressure gradient is insufficient to overcome surface tension and dislodge individual droplets from a pore region. This jamming phenomenon leads to a distinct difference between flows at constant pressure gradient and flows at constant flow rate (analogous to the difference between constant shear rate and constant stress in rheometric flows). Additional complications arise when compact droplets undergo break-up or coalescence leading to changing droplet size distributions. Greatly complicating the problem is the issue of wettability and dynamic contact lines moving over the highly irregular solid surface. To date, we still do not have true predictive capability for dynamic contact lines moving through even the most ideal systems.

\section{Overview}

Against this background, there are multiple avenues to pursue with useful results. In the petroleum industry, one accepts that relative permeability is an incomplete model which may not capture all of the relevant physics. Nonetheless, large-scale simulations become possible with multi-kilometre reservoir models incorporating large fluctuations in permeability, discontinuities associated with geological faults and mixtures of permeable and impermeable strata. At the opposite extreme, fluid mechanicians have studied simplified models in great detail, often leading to now classic phenomena in fluid mechanics. The Saffman-Taylor (Saffman \& Taylor 1958) instability or Hill instability (Homsy 1987), associated with viscous fingering in flow through porous media is one excellent example. Similarly, the Bretherton problem (Bretherton 1961) for a long droplet moving through a tight-fitting capillary with strong surface tension has yielded its own rich literature. Despite the intrinsic appeal of these fundamental studies, none exhibits the wealth of complex physical phenomena discussed above for multiphase flow in porous media. This suggests an intermediate path, made feasible by modern computational resources, which may shed some valuable light on the microscale dynamics and help to bridge the gap between proper resolution of microscale physics and useful macroscale approximations like relative permeability. This is the path followed by Zinchenko \& Davis (2013), (hereafter ZD). These authors do not attempt to capture all of the phenomena for flow in real media, most notably limiting attention to emulsions where the dispersed phase is non-wetting and thus avoiding the intractable issue of moving contact lines. In addition, they limit this work to liquids with equal viscosity; this feature reduces computational effort but is not otherwise an insurmountable obstacle. Finally, they restrict their simulations to the case of constant pressure gradient, which has some interesting implications for relative permeability compared to the case of constant flow rate. Despite these limitations, their 
(a)

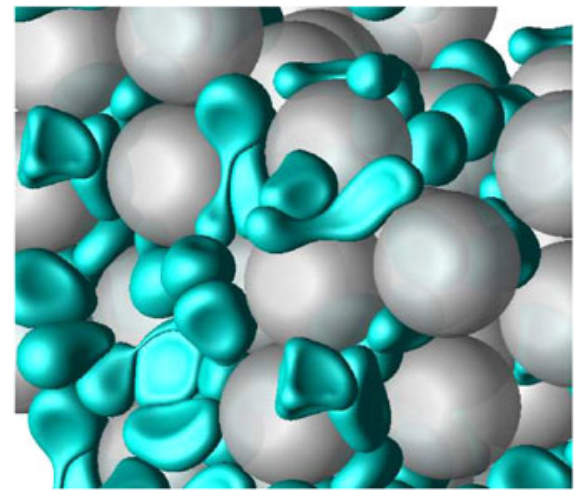

(b)

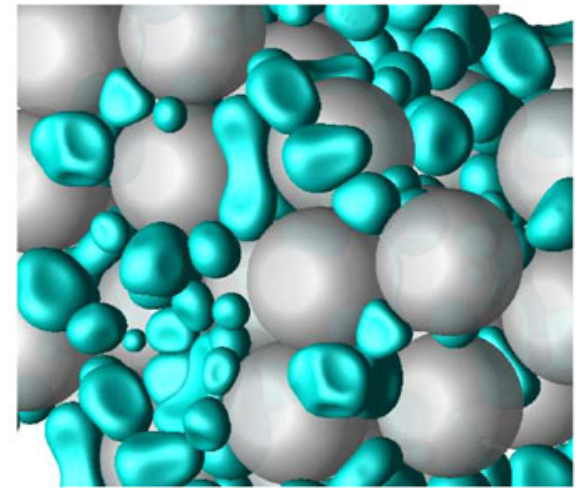

FIGURE 1. Pattern of droplets (blue-green colour) in emulsion at $C a=0.02$ before the first break-up $(a)$ and after multiple break-ups $(b)$ in flow through a packed bed of spheres (grey); from Zinchenko \& Davis (2013), figure 9.

effort significantly expands the scale and scope of computational work on multiphase flow through porous media.

One of the significant questions addressed in the featured paper is how the relative size of the mean droplet velocity $U_{D}$ and the mean velocity $U_{C}$ of the continuous phase vary as functions of the capillary number $C a$, the droplet size and the saturation. Relative permeability is usually defined for superficial velocity or volume flow rate which is found by multiplying $U_{D}$ or $U_{C}$ by the appropriate volume fraction. In their figure 6, ZD show that droplets in emulsions with tight-fitting droplets at small $C a=0.006$ tend to move faster than the continuous phase, though the velocities are generally within about $10 \%$. As $\mathrm{Ca}$ is increased, surface tension is weaker and droplets may experience one or more complex break-up sequences as illustrated here in figure 1 and in ZD figures 9 and 10. After due consideration of the break-up evolution, ZD show an interesting functionality for $U_{C}$ and $U_{D}$ as functions of $C a$ in figure 15. In all cases shown, the droplets move faster than the continuous phase, with the two velocities being nearly equal for the smallest $\mathrm{Ca}$. The interesting result is that highly deformable droplets at $C a=0.02$ after breaking into smaller droplets move almost $50 \%$ faster than the continuous phase. In addition, ZD note that the asymptotic mean velocities $U_{D}$ and $U_{C}$ are reached very early in the process, well before the avalanche of drop break-ups is concluded.

This surprising result may be explained by considering the effects of the droplet presence on the fluid velocity. Let $\boldsymbol{u}(\boldsymbol{x})=\boldsymbol{u}_{o}(\boldsymbol{x})+\boldsymbol{u}^{\prime}(\boldsymbol{x})$ be the velocity field throughout the pore space. Here $\boldsymbol{u}(\boldsymbol{x})$ is the total velocity in the fluid (either phase), $\boldsymbol{u}_{\boldsymbol{o}}(\boldsymbol{x})$ is the single-phase velocity field that would arise in the absence of all droplets and $\boldsymbol{u}^{\prime}(\boldsymbol{x})$ represents the perturbation to the velocity field due to the presence of the droplets. Note that $U_{D}$ and $U_{C}$ are weighted averages of $\boldsymbol{u}(\boldsymbol{x})$ restricted to the droplet volume and continuous-phase volume respectively. Changes in $U_{D}$ relative to $U_{C}$ may be attributed to two effects: changes in the perturbation velocity field $\boldsymbol{u}^{\prime}(\boldsymbol{x})$ and changes in the configuration of the averaging volumes. For the equal-viscosity, $C a=0.02$ multiple drop break-up runs in ZD, we speculate that $\boldsymbol{u}^{\prime}(\boldsymbol{x})$ has only a modest effect, and that the increased droplet velocity is due primarily to the biased sampling volume. This sampling volume does not change dramatically pre-break-up versus post-break-up; but in each case, it is heavily biased toward volumes with higher fluid velocity, thus 
leading to $U_{D}>U_{C}$. ZD point out two extensions to their results based on other simulations. The first is that stiffer droplets at still lower $C a$ will become trapped in the medium, and hence the droplet velocity will suddenly drop to zero at a critical $\mathrm{Ca}$. The second is that more viscous droplets with viscosity ratio $\lambda>1$ will consistently show a lower velocity than the continuous phase. These two cases are instances where the effects of the perturbation velocity $\boldsymbol{u}^{\prime}(\boldsymbol{x})$ become dominant leading to dramatically different behaviour.

In addition to the direct results given by $\mathrm{ZD}$, we may infer additional results for real porous media. If a system is operated under conditions of constant flow rate, a certain fraction of the pore space will become blocked by trapped droplets; however the flow will be channelled into more permeable channels through the medium. Since the overall flow rate is fixed, $\mathrm{Ca}$ in these unblocked channels will rise, and there will never be a critical jamming criterion. Local jamming may occur in regions of the medium, but the entire flow cross-section cannot be impermeable to droplet motion.

While the results above apply to concentrated emulsions, ZD also analyse the behaviour of dilute emulsions by studying the motion of a single droplet moving through the porous medium. Here there are three interesting results. The first is that single droplets are much less likely to break up than droplets in concentrated emulsions. The second is that single droplets travel several times faster than droplets in concentrated emulsions, moving at up to three times the speed of the continuous phase. Owing to the interference between droplets in the concentrated emulsion, this result is not surprising, but the quantitative size of the speed-up is unexpected. The third result is that large single droplets travel much faster than small single droplets. This last result is likely to be due to the bias in the averaging volume discussed above. While the perturbation velocity $\boldsymbol{u}^{\prime}(\boldsymbol{x})$ is not much changed, the sampling volume for larger droplets is more biased toward high-velocity regions in the flow. The validity of this hypothesis could easily be tested by auxiliary computations to evaluate directly the effect of $\boldsymbol{u}^{\prime}(\boldsymbol{x})$.

\section{Future}

The simulations presented by ZD have provided but a few illustrative samples of the phenomena which may arise in concentrated multiphase flow through porous media. The simulations constitute a significant advance in our ability to model multiphase flows, and efforts along these lines should be vigorously pursued. Owing to uncertainties in the physical assumptions and in the computational approximations however, these results should not be viewed as eliminating the need for detailed experimental results or more refined microscale analysis of individual phenomena.

\section{References}

Adler, P. M. \& Brenner, H. 1988 Multiphase flow in porous media. Annu. Rev. Fluid. Mech. 20, 35-59.

Bretherton, F. P. 1961 The motion of long bubbles in tubes. J. Fluid Mech. 10, 166-188.

Homsy, G. M. 1987 Viscous fingering in porous media. Annu. Rev. Fluid. Mech. 19, 271-311.

SAFFMAN, P. G. \& TAYLOR, G. I. 1958 The penetration of a fluid into a porous medium or Hele-Shaw cell containing a more viscous liquid. Proc. R. Soc. Lond. A 245, 312-329.

Wooding, R. A. \& Morel-SeytouX, H. J. 1976 Multiphase fluid flow through porous media. Annu. Rev. Fluid Mech. 8, 233-274.

Zinchenko, A. Z. \& DAVIS, R. H. 2013 Emulsion flow through a packed bed with multiple drop breakup. J. Fluid Mech. 725, 611-663. 